\title{
High-resolution solar imaging for citizen science
}

\author{
Alfred Yong Liang Tan ${ }^{1,2, *}$ \\ ${ }^{1}$ Elected Fellow, Institute of Physics, Singapore \\ ${ }^{2}$ National Junior College, 37 Hillcrest Road, Singapore
}

\begin{abstract}
High-resolution solar imaging, essential for research into the Sun is usually limited to high-end expensive telescopes found in professional observatories using adaptive optics technology. However, a Schmidt-Cassegrain Telescope (SCT) or a refractor telescope equipped with a narrow-band energy rejection filter and a suitable solar spectrum filter, employing appropriate data acquisition and post-processing techniques can provide an amateur astronomer a costeffective means to high-resolution solar imaging.
\end{abstract}

\section{Introduction}

In the last decade, students in Singapore schools have produced solar images in Hydrogen-Alpha, Calcium-K and White-light wavelengths. There was a keen interest to produce high-resolution solar images of the Sun for research work [1].

Students in several schools in Singapore conducted extensive tests from 2016 to 2019 to customise different solar telescope configurations and build a cost-effective observatory for producing high-resolution solar images [2, 3]. National Junior College used the findings of the trials and built a High-Resolution Solar High Observatory (HISRO). Following its commissioning in July 2019, students from NJC and other participating schools have used HISRO for their research work (See Figure 1).

\section{Equipment for High-resolution Imaging}

Different locally-customised configurations of high-resolution imaging rigs were tested. A typical configuration for high-resolution imaging is shown in Figure 2. The setup comprises:

- Dielectric-Energy Rejection Filter

- Host telescope

- Solar Spectrum Filter

- Astro camera with barlow or reducer

- German Equatorial Mount

- Field Imaging Instrumentation Console

\subsection{Dielectric-Energy Rejection Filter (D-ERF) [4]}

\footnotetext{
${ }^{*}$ Email : yltansg@gmail.com
} 
This project used D-ERFs with diameter ranging from $165 \mathrm{~mm}$ to $356 \mathrm{~mm}$ to enable the host telescope to retain its full-aperture resolving power while reducing the thermal energy going through the telescope to the solar spectrum filter.

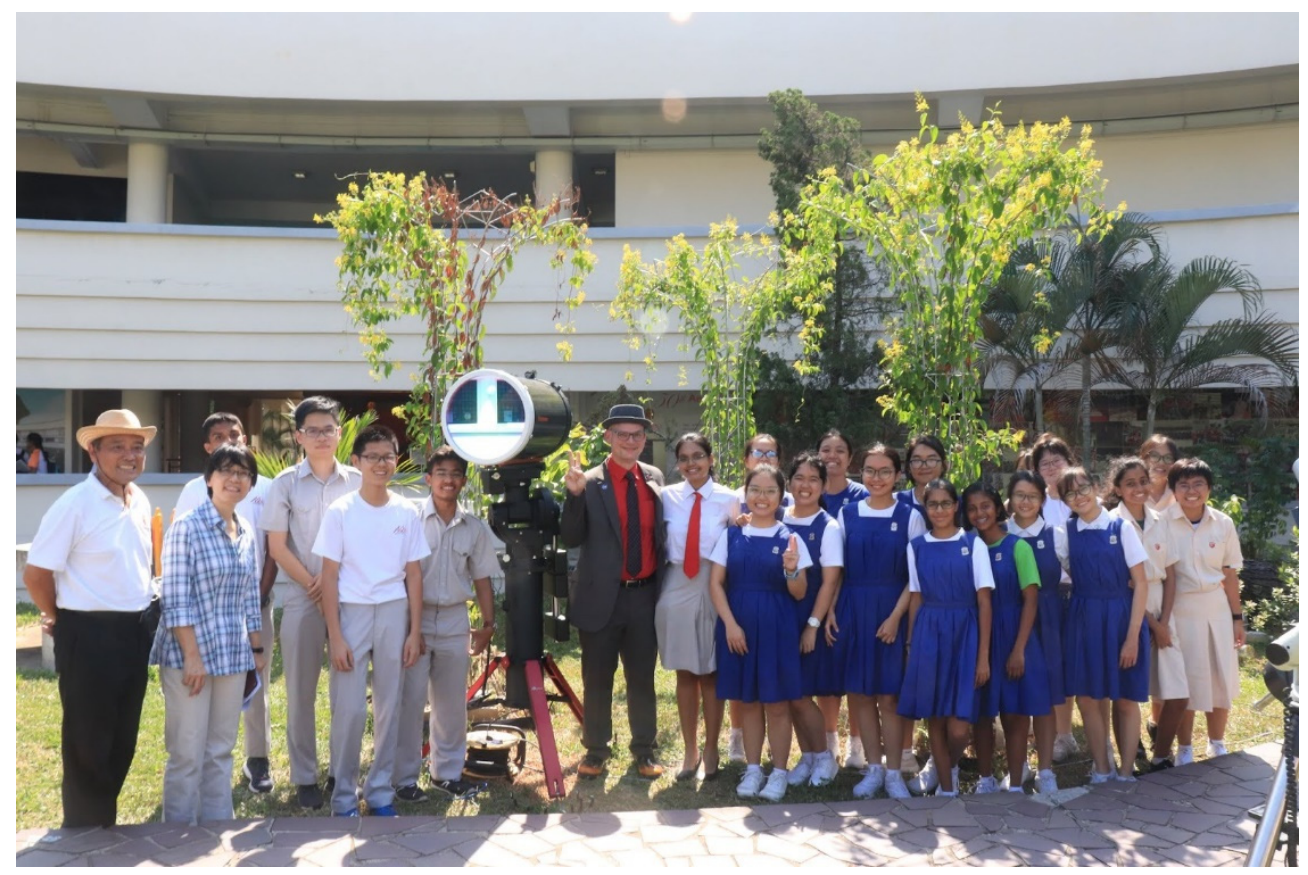

Fig. 1. HISRO was opened by Dr. C. Alex Young, NASA Goddard. 500 students from 12 schools attended the opening and solar observation on that day



Fig. 2. A typical setup for high-resolution solar imaging 
The D-ERF reduced the heat stress within the telescope and also reduced the thermally-induced seeing effects as well. A single-band H-Alpha $165 \mathrm{~mm}$ D-ERF was used on a $150 \mathrm{~mm}$ refractor telescope. A $295 \mathrm{~mm}$ D-ERF (for $279 \mathrm{~mm} \mathrm{SCT}$ ) and $214 \mathrm{~mm}$ D-ERF (for 203mm SCT) were used for tri-band (H-Alpha, Calcium-k and G-Band) imaging. A $365 \mathrm{~mm}$ D-ERF was used for H-Alpha imaging with a 356mm SCT. Typical H-Alpha tranmission and Calcium-K transmission were about $90 \%$ and $60 \%$ respectively (See Figure 3). "Leakage" into the unwanted wavlength was typically under $5 \%$.



Fig. 3. Four types of dielectric-Energy rejection filters (D-ERF) ranging from $165 \mathrm{~mm}$ to $356 \mathrm{~mm}$ were used on different-sized refractors and SCTs

\subsection{Host Telescope [5, 6]}

Typical SCTs, with SCT aperture ranging from $203 \mathrm{~mm}$ to $356 \mathrm{~mm}$ and a $150 \mathrm{~mm}$ refractor were used as host telescopes.

\subsection{Solar Spectrum Filter [5, 6]}

The project teams tested a range of commerically available etalons and integrated them into donor telescopes fitted with full-aperture D-ERF to form different customised solar telescope configurations. Favourite components included a solid mica etalon and air-spaced etalons without central obstruction for both H-Alpha and Calcium-k imaging. Other components included G-Band filter, H-Alpha blocking and Calcium-K module (See Figure 4).

\subsection{Astro camera with barlow or reducer.}

An astro CCD camera with a high acquisition speed was used for high-resolution solar imaging. To achieve the required imaging scale the necessary barlow or reducer was added before the astro camera.

\subsection{German Equatorial Mount.}


To provide smooth tracking of the Sun, a typical German Equatorial Mount with polar alignment was used to carry the solar telescope and imaging train.



Fig. 4. Different types of etalons were used to provide the desired bandpass for highresolution imaging

\subsection{Field Imaging Instrumentation Console.}

To have a dark workspace in the field, the team constructed a field imaging instrumentation console. The enclosure shielded the recording equipment from sunlight and enabled easy video acquisition in the field [7].

\section{Open field observatory [8]}

NJC High-resolution Solar Research Observatory has four imaging mounts holding three SCTs and one refractor telescope for high-resolution solar imaging. It was built with the following considerations:

- Open concept. It was located at a site for easy access for students to conduct solar observation and research work. (See Figure 5)

- Imaging environment. Seeing conditions affect the quality of imaging. Heated air from the ground will produce turbulence and affect the quality of the imaging. The team selected a site surrounded by greenery and situated on a relatively high ground.

- Setup time. Students attend lessons during daytime and they need to commence imaging as soon as possible when the seeing conditions permit highresolution imaging. HISRO was able to commence imaging within 5 minutes of reaching the observatory.

The team achieved the above-mentioned requirements by building HISRO at a location where students will walk past in between lectures. The telescopes were left 
permanently on the mounts and protected from the elements using a newly devised 3layer covering method (See Figure 6). Students are able to commence imaging after a 5-minute setup time. They have to remove the protective covers, deploy the field imaging instrumentation console and power up the telescopes before commencing the imaging session.

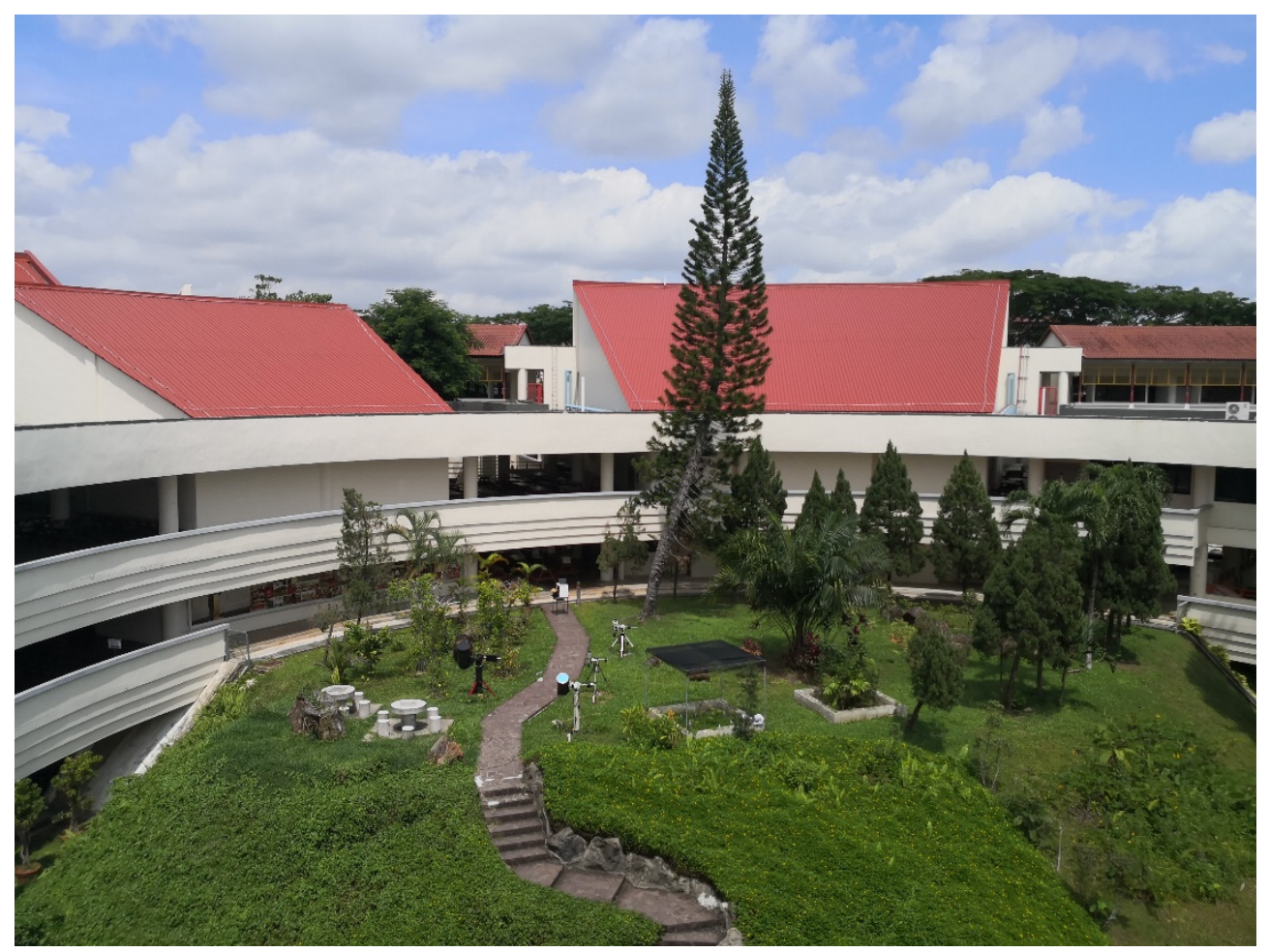

Fig. 5. Aerial view of National Junior College High-Resolution Solar Research Observatory 


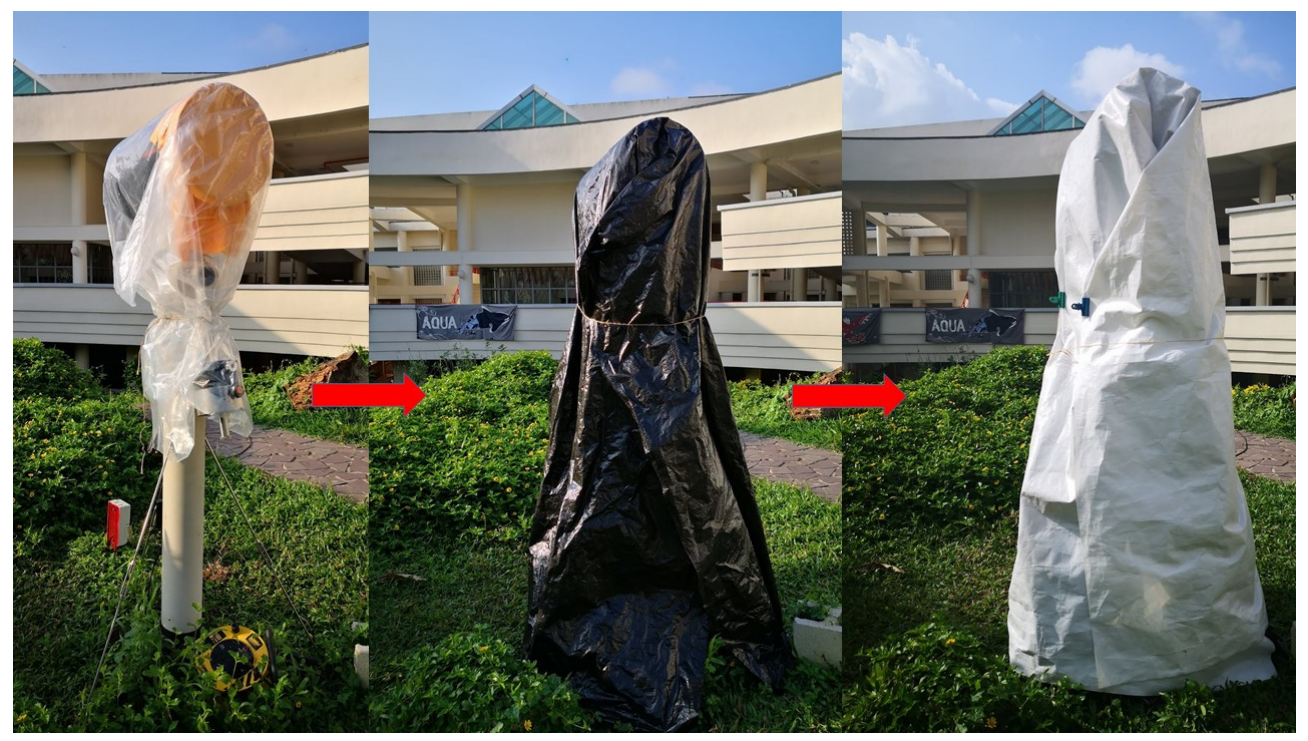

Fig. 6. Telescopes were left on the mount when not in use. A 3-layer covering method, devised by $\mathrm{Mr}$ A. Tan, provided protection of the equipment from the elements. This method of covering and protection has been trialed for a year. All the equipment did not show signs of deterioration in Singapore's humid and wet weather.

\section{Workflow}

Atmospheric turbulence randomly distorted the wavefronts emanating from the Sun and was the primary obstacle in achieving diffraction-limited resolution in high-resolution solar imaging. The following workflow was employed to reduce the adverse effect of atmospheric turbulence.

\subsection{Imaging window [9]}

The best quality images were acquired in the imaging window from 8:15 am to 10:30 am local time, throughout the year. This early window of opportunity will avoid jet stream emanating from the heated ground and rooftop. Nevertheless, the observatory was able to produce images of decent quality during the other times of the day due to the general good seeing condition in Singapore.

\subsection{Data acquisition [10]}

The camera has to be set up for each telescope configuration for optimum performance. To obtain individual captured frames with the best signal/noise ratio, the researcher needs to set the camera at the fastest shutter speeds with the least gain (brightness amplification) for least noise in the final image. Exposure was typically set below $5 \mathrm{msec}$ to freeze the seeing. The capture rate was usually maintained close to 100 fps to prevent a long duration capture leading to changes in the solar features. Gamma setting was set to provide good image contrast to aid the focussing of the image. During capture, gamma moderation was necessary to ensure that subtle solar details within 
dark regions were not lost. Typically each video capture will last about 30 seconds of about 2000 captured frames.

\subsection{Lucky imaging $[11,12]$}

Before image restoration techniques were applied, the frames captured in the most favourable atmospheric conditions (lucky frames) were selected using solar image processing software. During good seeing, up to 1,000 best frames were selected and stacked by the same solar image processing software to form a single resulting image with a high signal-to-noise ratio. There was a need to experiment and try stacking different number of selected frames to find the best resulting image.

\subsection{Post-processing [9]}

As part of image restoration, the final stacked image was further processed using Lucy Richardson deconvolution and unsharp masking techniques to produce a sharp and yet not overly sharpened high-resolution image revealing the fine features in the captured region of the Sun. The post-processed image could be further enhanced with an image editing software.

\section{Results}

\subsection{M5.0 flare.}

A hybrid solar telescope using a typical $150 \mathrm{~mm}$ achromatic refractor fitted with a full-

aperture D-ERF achieved an image scale of 0.45 arcsec per pixel (See Figure 7). It captured a M5.0 flare on 23 July 2016. (See Figure 8) The A\&G News \& Reviews in Astronomy \& Geophysics Volume 58, Issue 6 December 2017 featured these images as a contribution to citizen science [13]. (See animated sequence at https://tinyurl.com/M5-0-flare). 




Fig. 7. 150mm H-Alpha solar telescope



Fig. 8. Sprays and surges with the solar chromospheric network and fibril structures

\subsection{G-Band granulations.}

Figure 9 shows a 430nm G-Band image of granules at the top of the convection zone in a typical quiet photospheric region. Granule down to $1000 \mathrm{~km}$ can be observed. It was taken on 26 April 2017 with a $203 \mathrm{~mm}$ SCT fitted with a tri-band (H-Alpha, 
Calcium-K and G-band) full-aperture D-ERF, a commercial G-Band (filter passband 10 $\mathrm{nm}$ ) and $\mathrm{x} 5$ barlow. (See Figure 10) With good seeing, the imaging rig, with an image scale of 0.23 arc sec/pixel reached the diffraction limit for this wavelength and this telescope size.

Many amateur astronomers were amazed by what a typical $203 \mathrm{~mm}$ SCT could achieve. They celebrated this achievement and compared it favourably with a $1.6 \mathrm{~m}$ specialized solar telescope equipped with advanced adaptive optical system at the National Solar Observatory. This breakthrough sparked off a lively discussion amongst amateur astronomers [14].

Figure 11 shows a G-Band image of twin sunspots in a photospheric region. This image was taken later in the day after the Figure 9 image. The seeing condition had deteriorated and thin clouds veiled the Sun. The gain on the camera was stepped up and a more noisy image resulted. These 2 images (i.e. Figure 9 versus Figure 11) showed the vital part seeing condition played in the eventual quality of image. Subsequent trials showed that a bigger image scale should be used to improve the image quality when seeing has gone downhill.

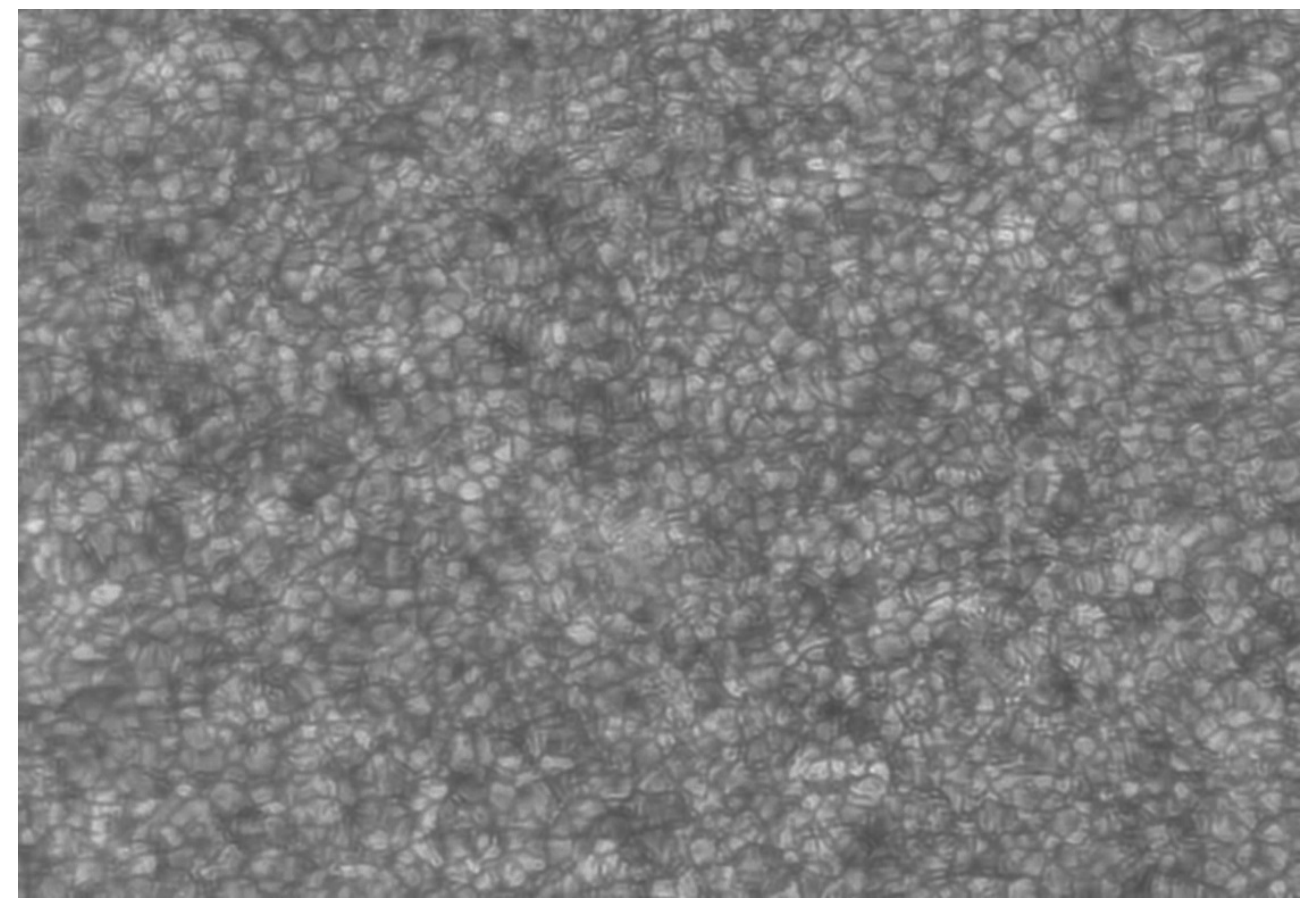

Fig. 9. A typical quiet photospheric region imaged by a $203 \mathrm{~mm}$ SCT in the G-band. Image scale was 0.23 arc sec per pixel. 


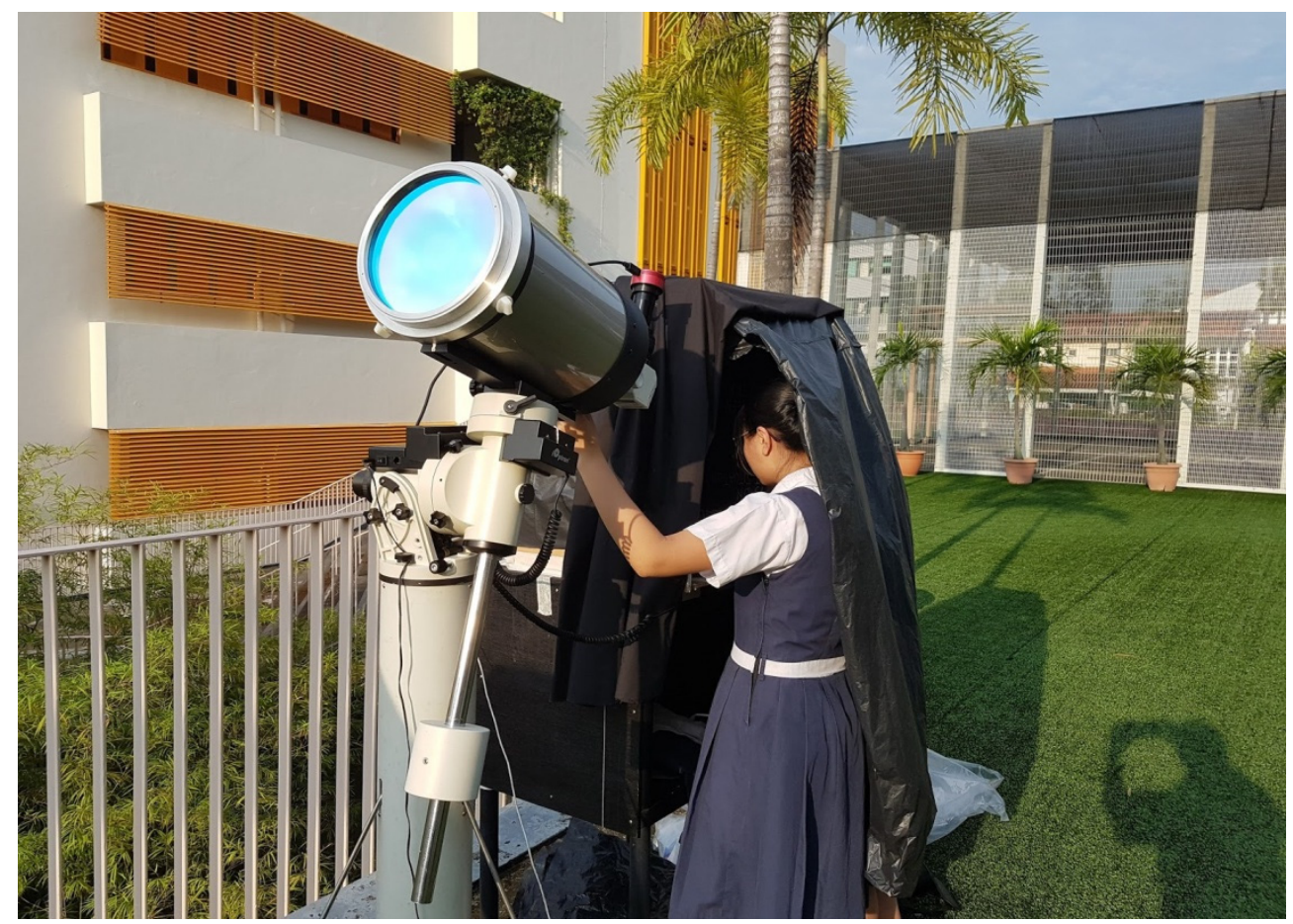

Fig. 10. G-Band imaging with a $203 \mathrm{~mm}$ SCT in Paya Lebar Methodist Girls' School

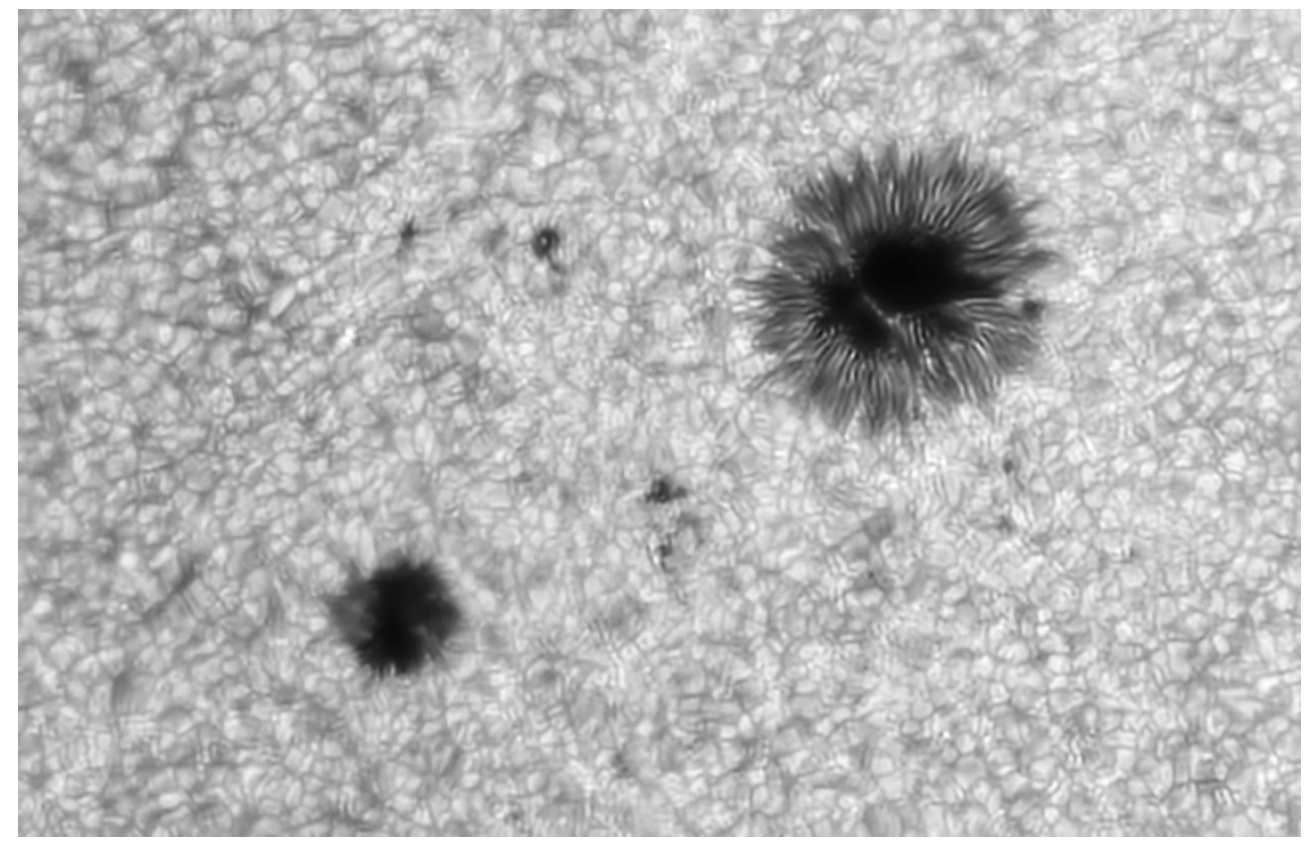

Fig. 11. G-band image of Sunspots in the photospheric regions. Image scale was 0.23 arcsec per pixel 


\subsection{Survey of the upper chromosphere during solar minima.}

A $279 \mathrm{~mm}$ SCT fitted with a tri-band (H-Alpha, Calcium-K and G-band) fullaperture D-ERF, a solid mica H-Alpha etalon and $\mathrm{x} 0.8$ reducer were used to survey the Sun's upper chromosphere in H-Alpha wavelength from January 2020 and February 2020 (See Figure 12).

Different sized reducers (e.g. x 0.5 to $x 0.8$ reducers) with different length spacers were used to achieve different image scale ranging from 0.15 to $0.2 \operatorname{arcsec}$ per pixel. Higher image scales were used in poorer seeing conditions.

Some of the collected images in Figures 13, 14 and 15 revealed fine fibril details, magnetic field topology and the dynamic nature of a quiet solar chromosphere [15]. As can be observed in Figure 14 and the evolution of fibrils could be traced. A boiling chromosphere was observed in an animated gif from a 5 minute recording session [16].

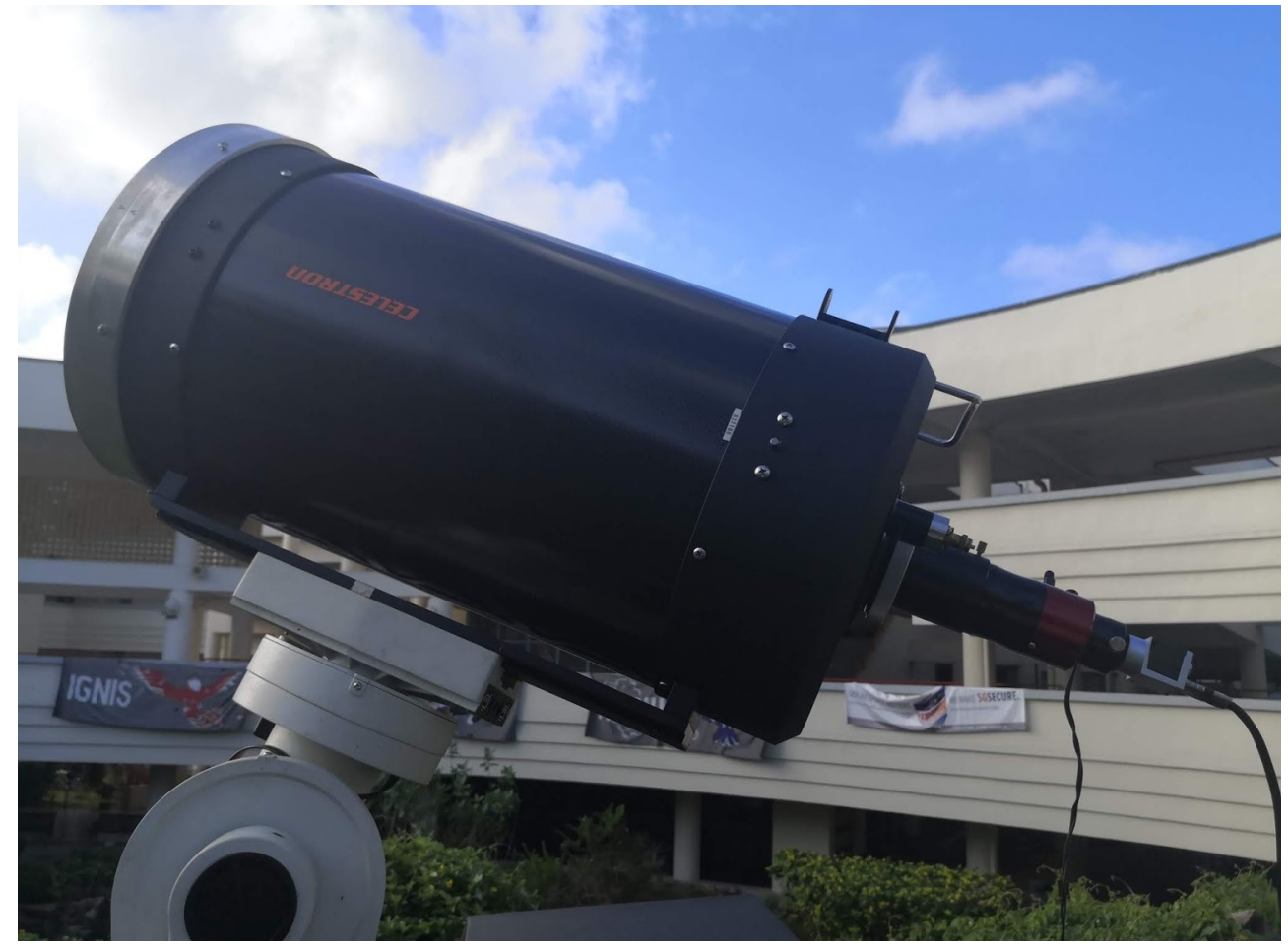

Fig. 12. A $279 \mathrm{~mm}$ SCT was used to survey the chromosphere with a H-Alpha mica etalon 


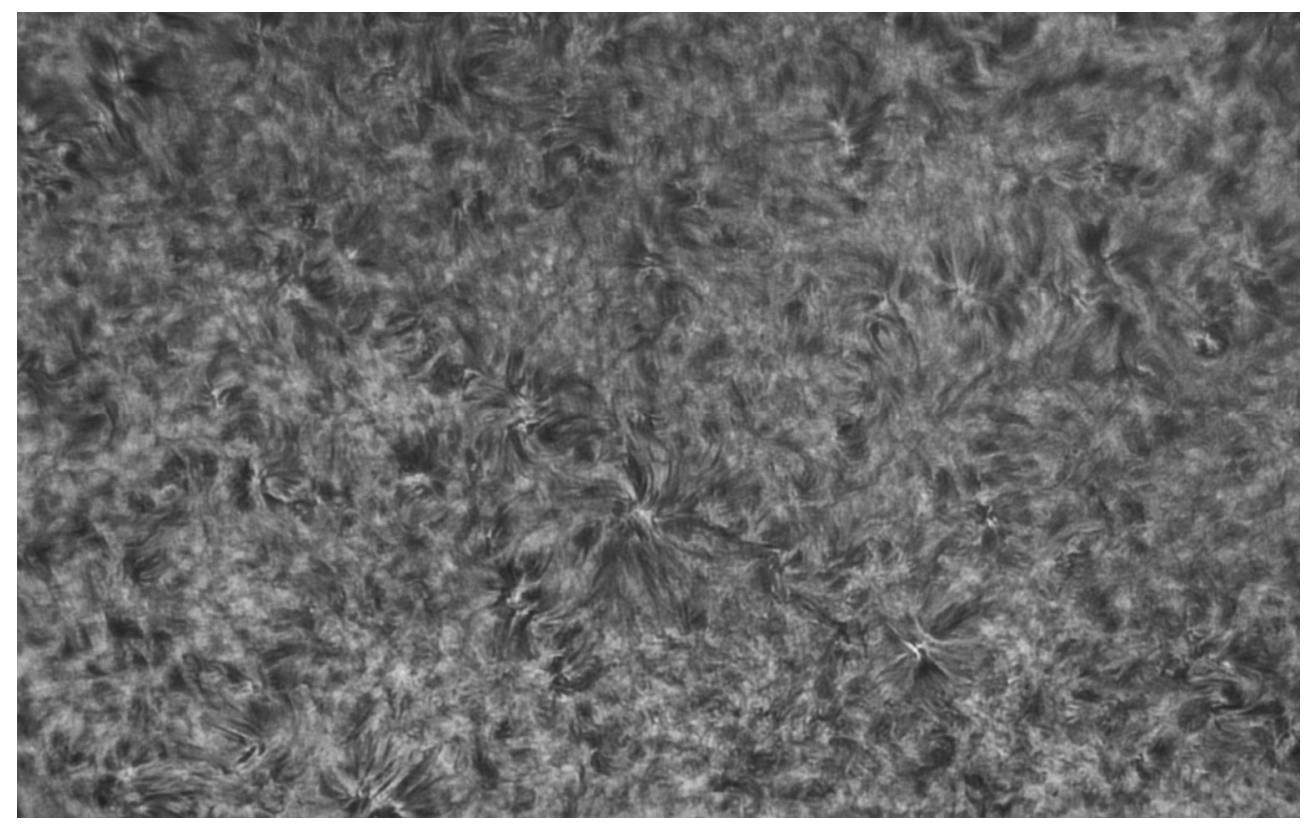

Fig. 13. Filamentary structures portray the magnetic field topology in the chromosphere

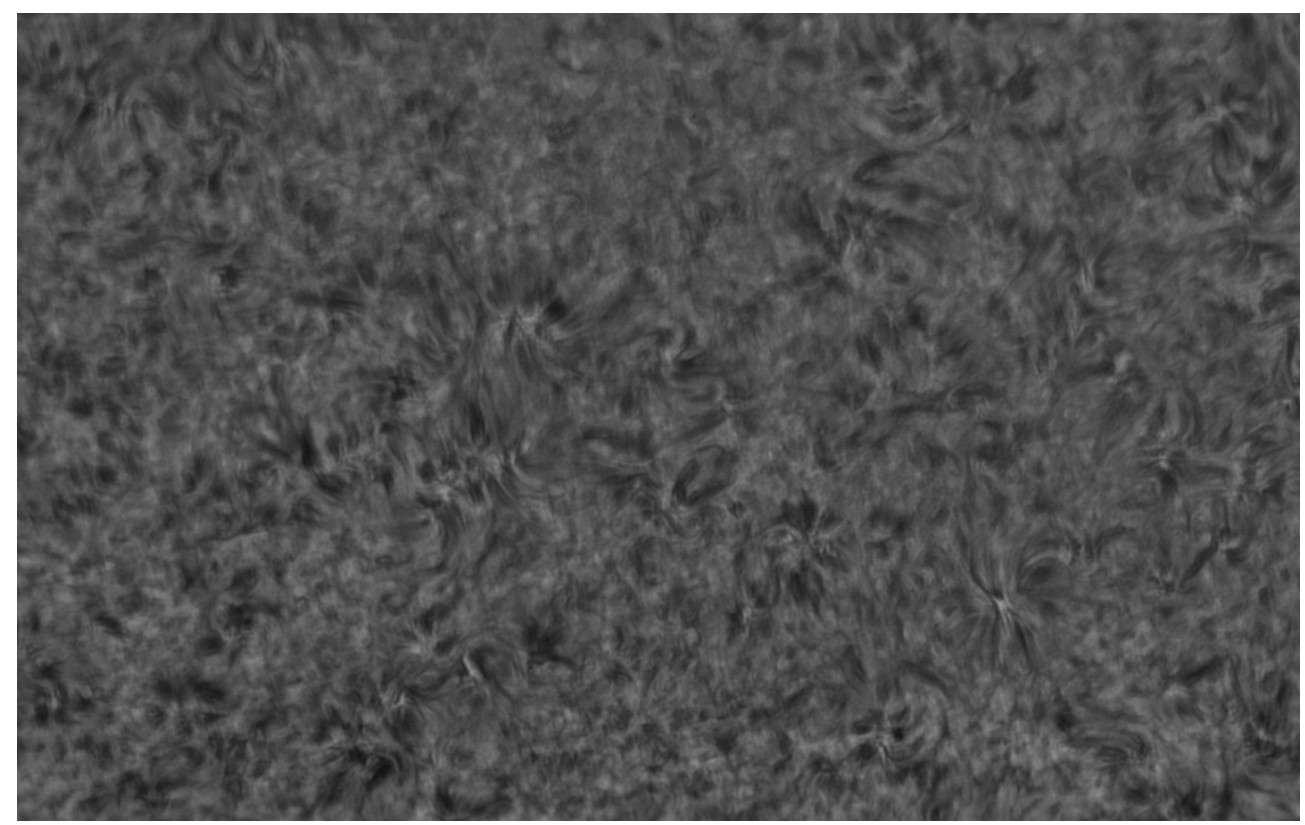

Fig. 14. A 5-minute animated gif showed the dynamic nature of a quiet solar chromosphere [16] 


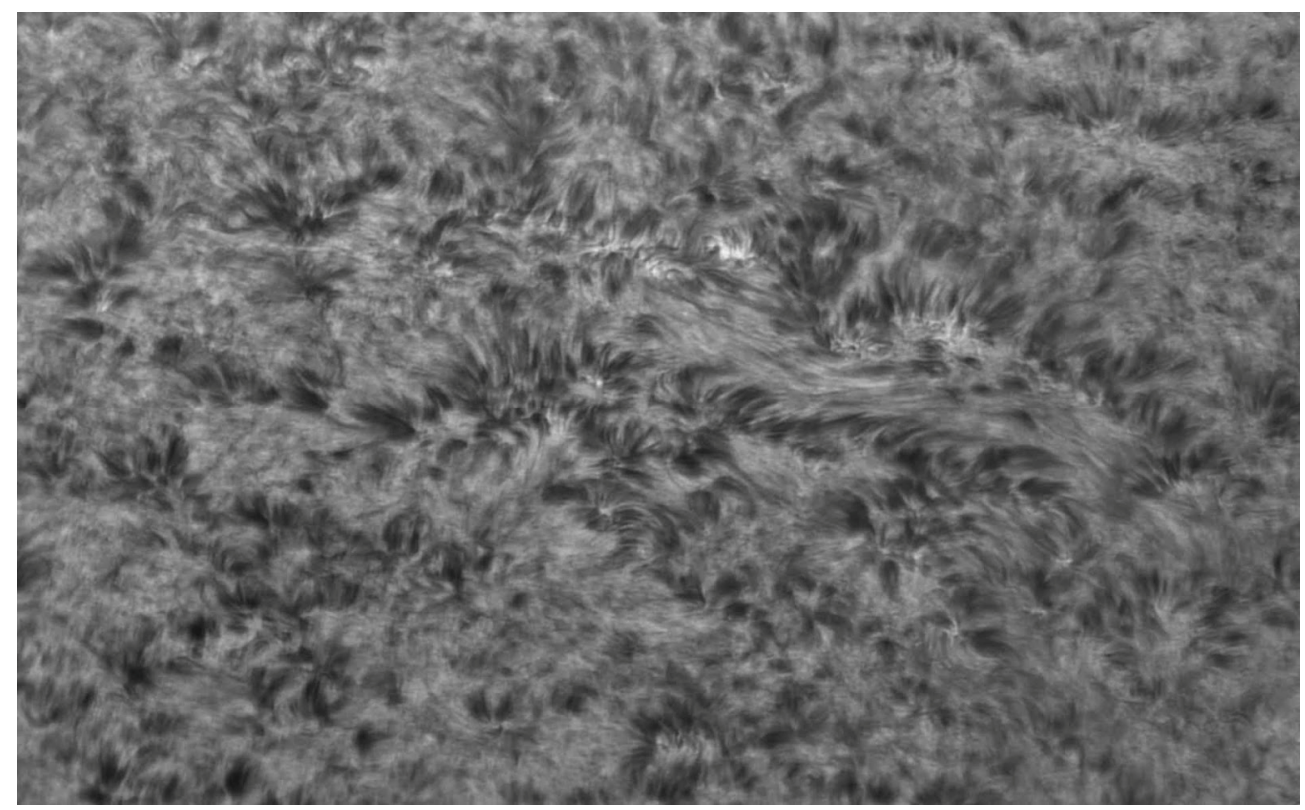

Fig. 15. Bright spots were frequently seen near multi layers of plasma fibrils

\subsection{Survey of the lower solar chromosphere}

The same $279 \mathrm{~mm}$ SCT fitted with a tri-band (H-Alpha, Calcium-K and G-band) full-aperture D-ERF was fitted with a calcium-K wedge for surveying the lower solar chromosphere. (See Figure 16). With a x2 barlow, an image scale of $0.22 \mathrm{arcsec}$ per pixel was achieved.

The chromospheric network as observed with this configuration are shown in Figure 17 and Figure 18 [17-19].

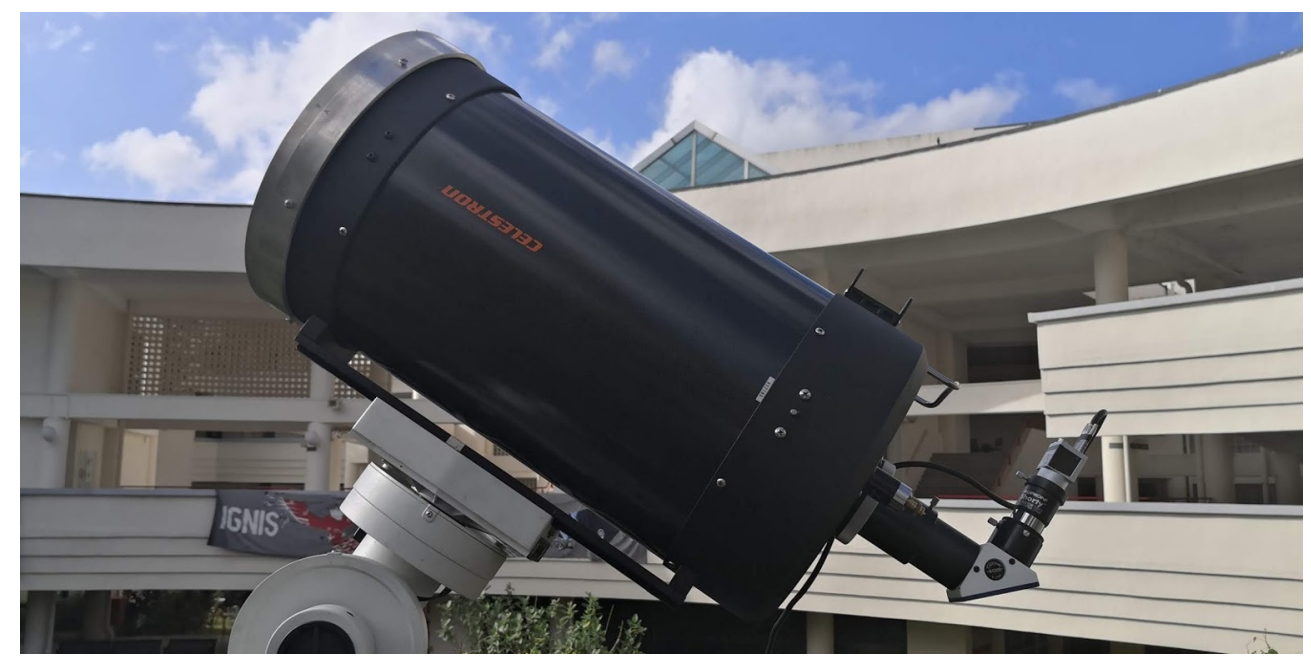

Fig. 16. A 279mm SCT fitted with a Calcium-K wedge was used to survey the lower solar chromosphere 


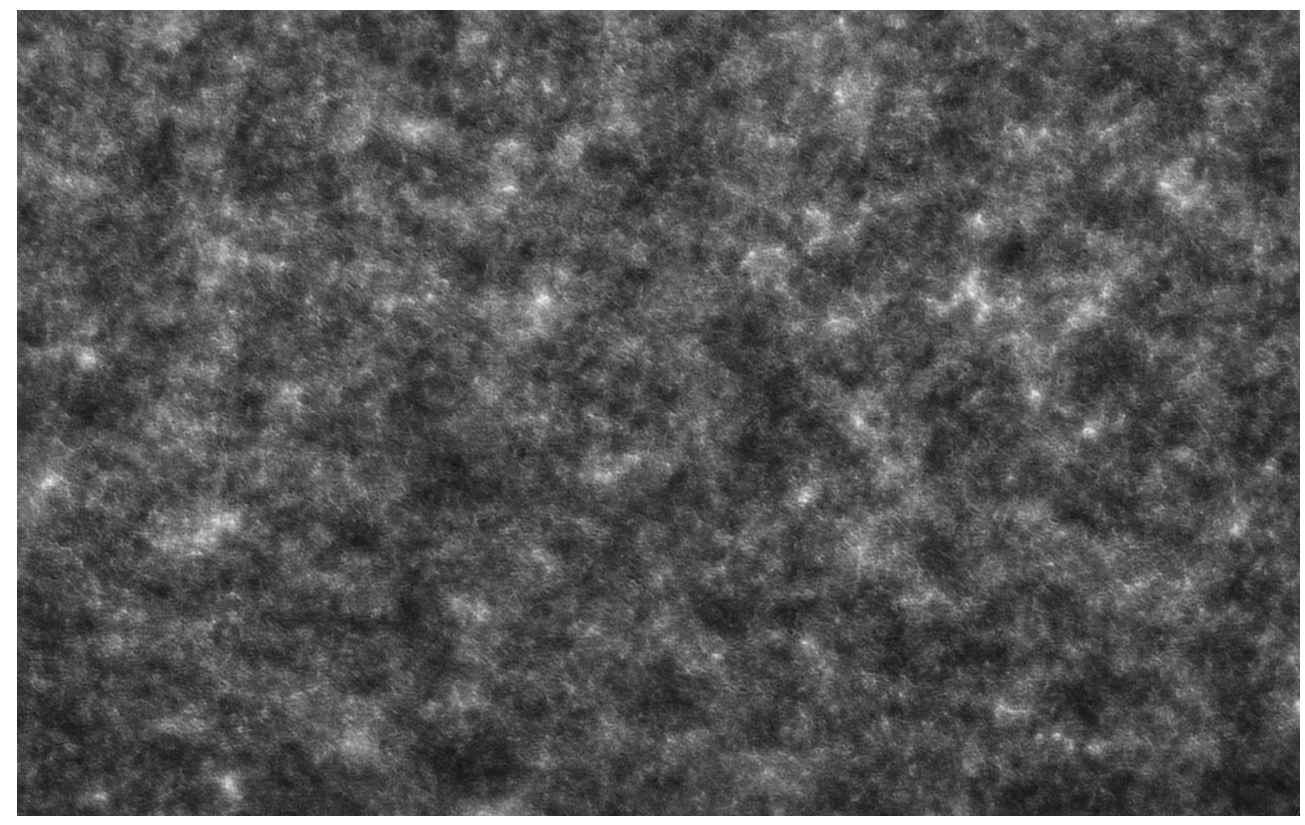

Fig. 17. Magnetic bright spots observed tend to concentrate in a granular pattern

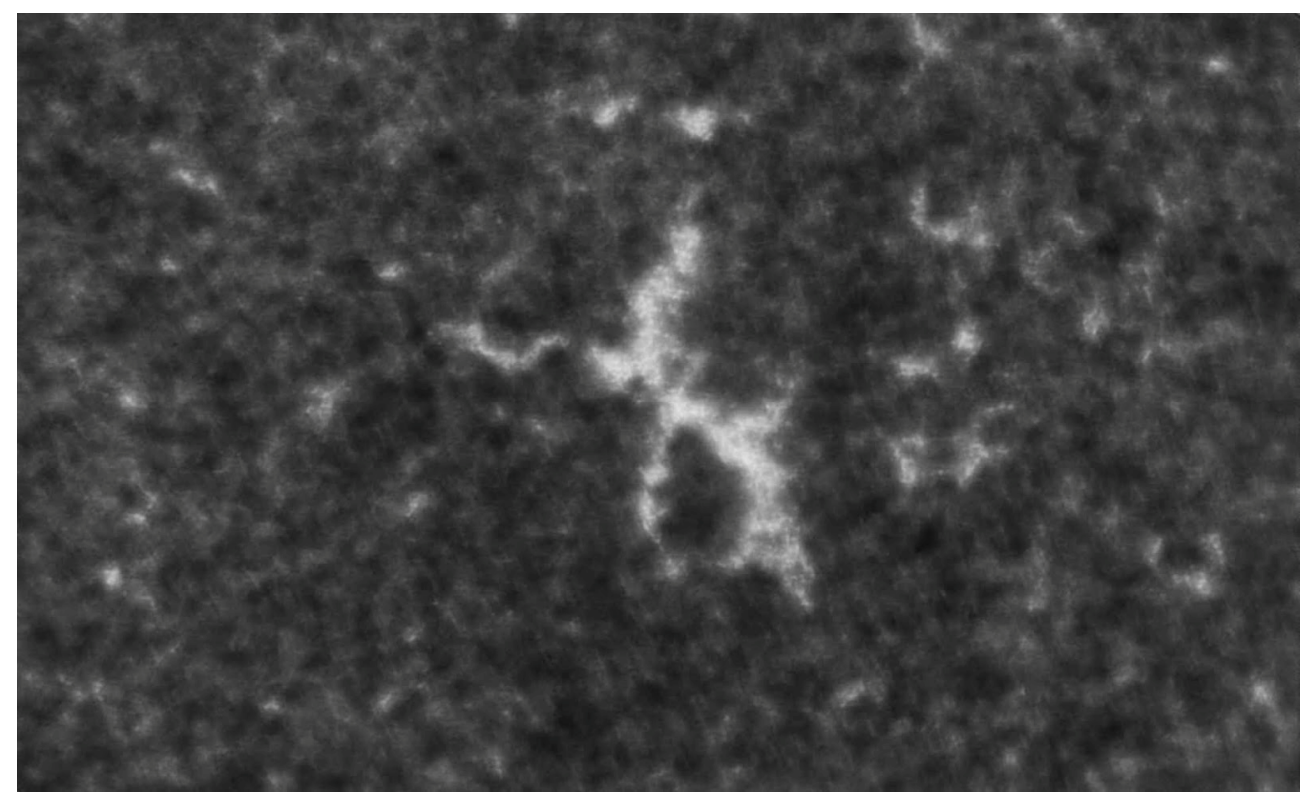

Fig. 18. The chromosphere network as observed with the 279mm SCT. Magnetic bright points formed a granular pattern.

\subsection{Fibrils}

The same $279 \mathrm{~mm}$ SCT shown in Figure 1 was fitted with a tri-band (H-Alpha, Calcium-K and G-band) full-aperture D-ERF and an air-spaced etalon without central obstruction to image the dark elongated fibril structures in H-Alpha wavelength [20]. 
Dark structures were observed in the penumbra of a sunspot, radically expanding around the umbra and creating filamentary structure in the active region (See Figure 19).

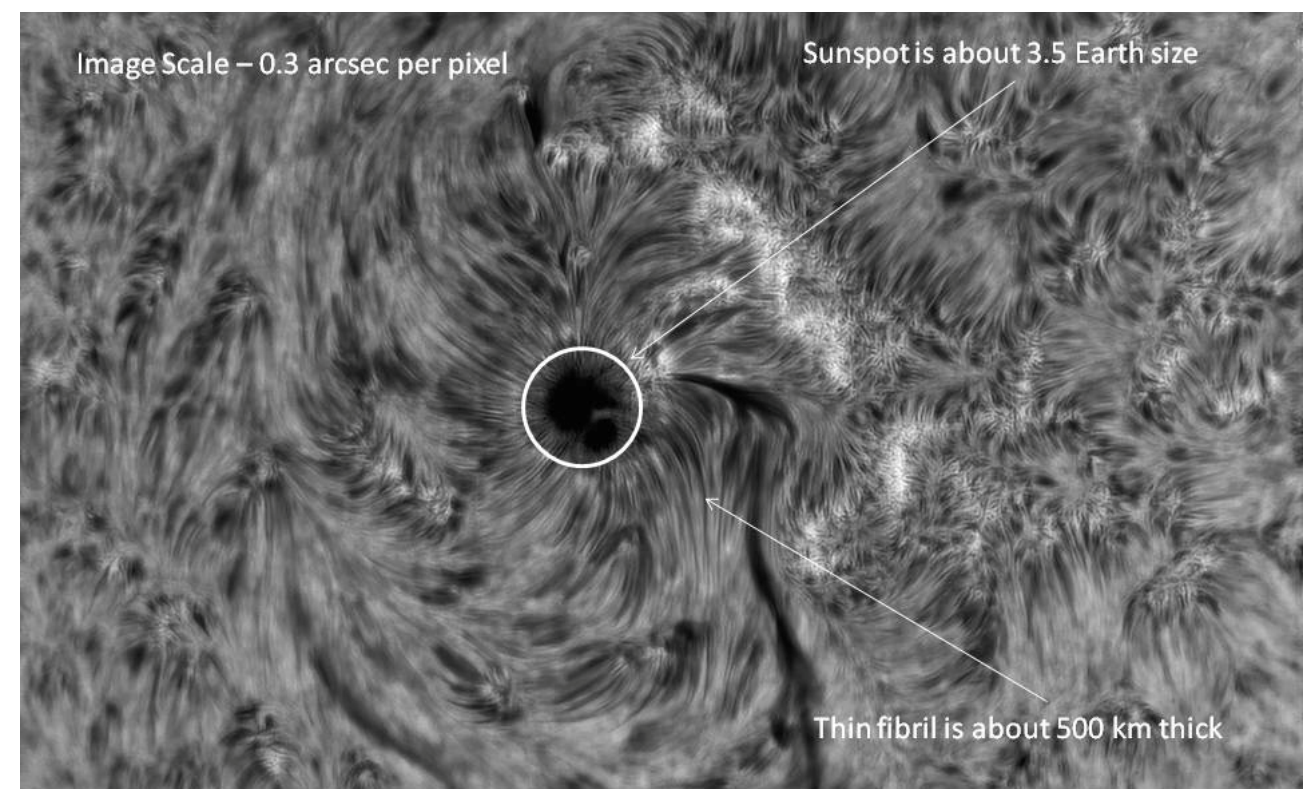

Fig. 19. Dark elongated fibril structures surround the sunspot

\section{Conclusion}

The different solar telescopes, when used with appropriate data acquisition, postprocessing and image restoration workflow, showed great potential as cost-effective solutions for amateurs to do high-resolution solar research. More work was needed to overcome atmospheric seeing when imaging with a $356 \mathrm{~mm}$ SCT and improving image restoration work processes to produce better quality images [21].

\section{Acknowledgments}

This material was a consolidation of the investigative work to establish different configurations of optimized high-resolution solar telescopes for different imaging requirements and to build an open solar observatory for students' research. Students and teachers from Paya Lebar Methodist Girls' School, National Junior College and other partner schools participated in this effort. They used the prototype telescopes for their respective teaching and learning activities. The images that they have produced provided valuable feedback for the project. I acknowledge the expert advice provided by fellow astronomers from https://solarchatforum.com/. I also acknowledge the support given by my wife, Mei for the purchase of all the equipment, encouraging me to press on since I began the project in 2016 and proof reading this material. 


\section{References}

1. Research activities for amateurs, http://www.astrosurf.com/luxorion/research.htm, (2020)

2. G. Garcia, ALPO Feature: The Sun High Resolution Solar Imaging, The Strolling Astronomer, 45, No.3, 16 -20, (2003)

3. H. Wang, C. Liu, Signatures of magnetic flux ropes in the low solar atmosphere observed in high resolution, Frontiers in Astronomy and Space Sciences, (2019)

4. Description of Airylab 235mm DERF, http://astrograph.net/Airylab-235mm-D-ERFFilter-including-Custom-Cell, (2020)

5. https://airylab.com/hat-solar-telescopes, (2020)

6. https://www.skyatnightmagazine.com/reviews/telescopes/airylab-203mm-hydrogenalpha-chromosphere-telescope/, (2020)

7. https://petapixel.com/2015/05/19/a-6-ikea-storage-box-makes-for-a-thriftycollapsible-laptop-sun-shade/, (2020)

8. https://www.nytimes.com/2002/07/18/garden/design-notebook-in-backyards-starryappeal.html, (2020)

9. https://solarnutcase.livejournal.com/18572.html, (2020)

10. K. M. Harrison, Camera exposure times, Imaging Sunlight Using a Digital Spectroheliograph (2016) Springer International Publishing, pp. 123

11. Law, N.M, Mackay, C.D.,Baldwin, J.E., Lucky imaging: High angular resolution imaging in the visible from the ground, (2006)

12. Beckers, J.M., Improving solar image quality by image selection. (1989)

13. L. Fletcher, A. Berlicki, A. K. Awasthi and D. Gronkiewicz, Recruiting flare hunters for citizen science, The A\&G News \& Reviews in Astronomy \& Geophysics, 58, Issue 6 December 2017

14. https://solarchatforum.com/viewtopic.php?f=4\&t=22409, (2017)

15. G.Tsiropoula, K. Tziotziou, I. Kontogiannis, M.S. Madjarska, J.G. Doyle, Y. Suematsu, , Solar fine-scale structure. pp. 8-10, (2012)

16. https://1.bp.blogspot.com/-K8-cR4Irj9M/XigpQYpdG5I/AAAAAAAApEE/DmhRdrHXcYDJWXrj56rtell-hhX8_dMACLcBGAsYHQ/s1600/10frames.gif

17. J. Leenaarts, M. Carlsson, L.R. Van Der Voort, On fibrils and field lines: The nature of Ha fibrils in the solar chromosphere, (2018)

18. V. H.Hansteen, M. Carlsoon, The solar atmosphere, pp 13-16, (2019)

19. J.L. Jenkins, Observing the Sun, Lower chromosphere, K-Grains, Chromospheric network, pp. 50 - 52, (2013) 
20. https://www.seeker.com/fibril-flowers-on-suns-surface-photo-1769556565.html (2015)

21. A. Macrobert, Sky\&Telescope, How to successfully beat atmospheric seeing, https://skyandtelescope.org/astronomy-equipment/beating-the-seeing/ (2006) 